\title{
Counterflow of spontaneous mass currents in trapped spin-orbit-coupled Fermi gases
}

\author{
E. Doko, ${ }^{1}$ A. L. Subaşı, ${ }^{2}$ and M. Iskin ${ }^{1}$ \\ ${ }^{1}$ Department of Physics, Koç University, Rumelifeneri Yolu, 34450 Sartyer, Istanbul, Turkey \\ ${ }^{2}$ Department of Physics, Faculty of Science and Letters, Istanbul Technical University, 34469 Maslak, Istanbul, Turkey
}

(Received 20 December 2011; published 24 May 2012)

\begin{abstract}
We use the Bogoliubov-de Gennes formalism and study the ground-state phases of trapped spin-orbit-coupled Fermi gases in two dimensions. Our main finding is that the presence of a symmetric (Rashba-type) spin-orbit coupling spontaneously induces counterflowing mass currents in the vicinity of the trap edge, i.e., $\uparrow$ and $\downarrow$ particles circulate in opposite directions with equal speed. These currents flow even in noninteracting systems, but their strength decreases toward the molecular Bose-Einstein-condensate limit, which can be achieved by increasing either the spin-orbit coupling or the interaction strength. These currents are also quite robust against the effects of asymmetric spin-orbit couplings in the $x$ and $y$ directions, gradually reducing to zero as the spin-orbit coupling becomes one dimensional. We compare our results with those of chiral $p$-wave superfluids and superconductors.
\end{abstract}

DOI: 10.1103/PhysRevA.85.053634

PACS number(s): 67.85.-d, 03.75.Hh, 03.75.Ss, 05.30.Fk

\section{INTRODUCTION}

The controversy over the expectation value of the intrinsic ground-state angular momentum of the $A$ phase of superfluid ${ }^{3} \mathrm{He}$ in a given container has a long history, and it has not yet attained a universally agreed resolution [1]. For $N$ weakly interacting particles, theoretical predictions for the BCS ground state vary by orders of magnitude from $N|\Delta| \hbar /\left(2 \varepsilon_{F}\right)$ to $N \hbar / 2$, where $|\Delta|$ is the amplitude of the order parameter, i.e., the pairing energy, and $\varepsilon_{F}$ is the Fermi energy. At a first glance, the former expectation, which is based on the observation that pairing affects not all but only a small fraction $\left(|\Delta| / \varepsilon_{F}\right) N$ of fermions, seems more intuitive. However, this subject has recently regained interest in the condensed-matter and atomic and molecular physics communities due to its relevance to the possible $p_{x}+i p_{y}$ superconducting phase of $\mathrm{Sr}_{2} \mathrm{RuO}_{4}$ [2] and atomic chiral $p$-wave superfluids [3], respectively, which support the latter expectation [4,5]. On one hand, these recent results seem intuitive at least in the strong-fermion-attraction limit of tightly bound $p$-wave Cooper molecules, where all of the molecules can undergo Bose-Einstein condensation (BEC) at zero temperature with each molecule having a microscopic angular momentum $\hbar$. On the other hand, they seem counterintuitive in the weak-fermion-attraction limit of loosely bound and largely overlapping $p$-wave Cooper pairs, since these results imply that the angular momentum in the BCS ground state is macroscopically different from its vanishing value in the normal ground state, when the pairing energy becomes arbitrarily small. While this controversy still awaits an experimental resolution, here we propose an alternative atomic system where the microscopic angular momentum of Cooper pairs can also give rise to a macroscopic one. With their unique advantages over condensed-matter systems, it is plausible that the macroscopic angular momentum of this alternative system may be observed for the first time with cold atoms, and that this would also shed some light on the ${ }^{3} \mathrm{He}$ controversy for which the basic mechanism is found to be similar.

Cold atom systems have already proved to be versatile in simulating various many-body problems. For instance, one of the major achievements with atomic Fermi gases in the past decade is the unprecedented realization of the BCS-BEC crossover [6], where the ground state of the system can be continuously tuned from the BCS to the BEC limit as a function of increasing fermion attraction with the turn of a knob. The main difference between the BCS-BEC crossover and the BCS theory is that in the crossover Cooper pairing is allowed not only for fermions with energies close to the Fermi energy but also for all pairs with appropriate momenta $[1,6]$. Having established the basic tools, it is arguable that one of the most promising research directions to pursue with cold Fermi gases is the artificial spin-orbit gauge fields. Such a field has already been realized with cold Bose gases [7,8] using a novel technique based on spatially dependent optical coupling between internal states of the atoms, and the same technique is equally applicable for neutral fermionic atoms. In fact, we have recently learned that the first generation of quantum degenerate spin-orbit-coupled Fermi gases has been created with ${ }^{40} \mathrm{~K}$ atoms [9].

This immediate possibility of creating a spin-orbit-coupled Fermi gas has already received tremendous theoretical interest in the condensed-matter and atomic physics communities: a number of exotic superfluid phases with balanced or imbalanced populations, at zero or finite temperatures, in two or three dimensions, etc., have been explored [10-23]. Motivated by these recent advances, here we study the ground-state and finite-temperature phases of trapped spin-orbit-coupled Fermi gases, and our main results are as follows. We find that the presence of spin-orbit coupling (SOC) spontaneously induces counterflowing $\uparrow$ and $\downarrow$ mass currents in the vicinity of the trap edge [24]. We show that these currents flow even in noninteracting systems, and that they are quite robust against the effects of asymmetric SOC in the $x$ and $y$ directions, gradually reducing to zero as the SOC becomes one dimensional. However, their strength decreases toward the molecular BEC limit, which can be achieved by increasing either the SOC or the interaction strength. We argue that the origin of spontaneously induced counterflowing mass currents in spin-orbit-coupled Fermi gases can be understood via a direct correspondence with chiral $p$-wave superfluids and superconductors, but with some fundamental differences.

The rest of the paper is organized as follows. In Sec. II, first we introduce the mean-field Hamiltonian that is used to describe the spin-orbit-coupled Fermi gases on optical lattices 
and then use the Bogoliubov-de Gennes (BdG) formalism to obtain generalized self-consistency equations for the number of fermions, superfluid order parameter, and strength of circulating mass currents. The numerical solutions of this formalism are presented in Sec. III, which is followed by a brief discussion of their experimental realization in Sec. IV. We summarize our main findings in Sec. V.

\section{THEORETICAL BACKGROUND}

In this paper, we consider a harmonically trapped twodimensional optical lattice in the presence of a non-Abelian gauge potential. In addition to the usual spin-conserving hopping terms, the main effect of such a gauge potential is the appearance of an additional spin-flipping hopping term in the single-particle kinetic energy term, as discussed next.

\section{A. Hamiltonian}

Spin-orbit-coupled Fermi gases on optical lattices can be described by the grand-canonical mean-field Hamiltonian

$$
\begin{aligned}
H= & -t \sum_{i, \hat{e}}\left(C_{i+\hat{e}}^{\dagger} \phi_{i+\hat{e}, i} C_{i}+\text { H.c. }\right) \\
& -\sum_{i}\left(\mu_{\sigma}+V_{\sigma i}^{H}-V_{i}^{T}\right) c_{\sigma i}^{\dagger} c_{\sigma i} \\
& +\sum_{i}\left(\Delta_{i} c_{\uparrow i}^{\dagger} c_{\downarrow i}^{\dagger}+\Delta_{i}^{*} c_{\downarrow i} c_{\uparrow i}\right),
\end{aligned}
$$

where the operator $c_{\sigma i}^{\dagger}\left(c_{\sigma i}\right)$ creates (annihilates) a pseudospin $\sigma=\{\uparrow, \downarrow\}$ fermion at lattice site $i$, the spinor $C_{i}^{\dagger}=\left(c_{\uparrow i}^{\dagger}, c_{\downarrow i}^{\dagger}\right)$ denotes the fermion operators collectively, $\hat{e}=\{\hat{x}, \hat{y}\}$ allows only nearest-neighbor hopping, and H.c. is the Hermitian conjugate. For a generic gauge field $\mathbf{A}=\left(\alpha \sigma_{y},-\beta \sigma_{x}\right)$, where $\sigma_{e}$ is the Pauli matrix and $\{\alpha, \beta\} \geqslant 0$ are independent parameters characterizing both the strength and the symmetry of the spinorbit coupling, the $\uparrow$ and $\downarrow$ fermions gain $\phi_{i+\hat{x}, i}=e^{-i \alpha \sigma_{y}}$ phase factors for hopping in the positive $x$ direction and $\phi_{i+\hat{y}, i}=$ $e^{i \beta \sigma_{x}}$ phase factors for hopping in the positive $y$ direction. Note that the spin-conserving and spin-flipping hopping terms are proportional, respectively, to $\cos \alpha$ and $\sin \alpha$ in the $x$ direction and to $\cos \beta$ and $\sin \beta$ in the $y$ direction. The off-diagonal coupling $\Delta_{i}$ is the mean-field superfluid order parameter to be specified below, and $V_{\sigma i}^{H}=g n_{-\sigma i}$ is the Hartree term, where $g \geqslant 0$ is the strength of the on-site attractive interaction and $n_{\sigma i}$ is the filling of $\sigma$ fermions at site $i$. Here, $(-\uparrow)=\downarrow$ and vice versa. We introduce $\sigma$-dependent chemical potentials $\mu_{\sigma}$ to fix the number of $\sigma$ fermions independently, but we assume both $\uparrow$ and $\downarrow$ fermions experience the same trapping potential $V_{i}^{T}=V_{0} r_{i}^{2} / 2$, where the distance $r_{i}$ is measured from the center in our $L \times L$ lattice. Next, we solve this Hamiltonian via the $\mathrm{BdG}$ formalism.

\section{B. Bogoliubov-de Gennes formalism}

The BdG equations are obtained by diagonalizing the quadratic Hamiltonian given above via a generalized Bogoliubov-Valatin transformation, leading to a $4 L^{2} \times 4 L^{2}$ matrix-eigenvalue problem,

$$
\sum_{j}\left(\begin{array}{cccc}
T_{\uparrow \uparrow} & T_{\uparrow \downarrow} & 0 & \Delta \\
T_{\downarrow \uparrow} & T_{\downarrow \downarrow} & -\Delta & 0 \\
0 & -\Delta^{*} & -T_{\uparrow \uparrow}^{*} & -T_{\uparrow \downarrow}^{*} \\
\Delta^{*} & 0 & -T_{\downarrow \uparrow}^{*} & -T_{\downarrow \downarrow}^{*}
\end{array}\right)_{i j}\left(\begin{array}{c}
u_{n j}^{\uparrow} \\
u_{n j}^{\downarrow} \\
v_{n j}^{\uparrow} \\
v_{n j}^{\downarrow}
\end{array}\right)=\varepsilon_{n}\left(\begin{array}{c}
u_{n i}^{\uparrow} \\
u_{n i}^{\downarrow} \\
v_{n i}^{\uparrow} \\
v_{n i}^{\downarrow}
\end{array}\right),
$$

where $u_{n i}^{\sigma}$ and $v_{n i}^{\sigma}$ are the components of the $n$th quasiparticle's wave function at site $i$, and $\varepsilon_{n} \geqslant 0$ is the corresponding energy eigenvalue. While the off-diagonal couplings are $\Delta_{i j}=\Delta_{i} \delta_{i j}$ diagonal in site indices since we consider on-site interactions only, the nearest-neighbor hopping and on-site energy terms can be written compactly as $T_{\sigma \sigma^{\prime}}^{i j}=-t_{\sigma \sigma^{\prime}}^{i j}-$ $\left(\mu_{\sigma}+V_{\sigma i}^{H}-V_{i}^{T}\right) \delta_{i j} \delta_{\sigma \sigma^{\prime}}$, where $\delta_{i j}$ is the Kronecker delta. Here, the nonvanishing nearest-neighbor hopping elements are $t_{\sigma \sigma}^{i, i+\hat{x}}=t \cos \alpha$ and $t_{\uparrow \downarrow}^{i, i+\hat{x}}=-t_{\downarrow \uparrow}^{i, i+\hat{x}}=-t \sin \alpha$ for the positive $x$ direction and $t_{\sigma \sigma}^{i, i+\hat{y}}=t \cos \beta$ and $t_{\uparrow \downarrow}^{i, i+\hat{y}}=t_{\downarrow \uparrow}^{i, i+\hat{y}}=i t \sin \beta$ for the positive $y$ direction. Note that the hoppings in the negative $x$ and $y$ directions are simply Hermitian conjugates.

Equation (2) needs to be solved simultaneously with the order parameter $\Delta_{i}=g\left\langle c_{\uparrow i} c_{\downarrow i}\right\rangle$ and number $n_{\sigma i}=\left\langle c_{\sigma i}^{\dagger} c_{\sigma i}\right\rangle$ equations for a self-consistent set of $\Delta_{i}$ and $\mu_{\sigma}$ solutions. Here, $\langle\cdots\rangle$ is a thermal average. Using the Bogoliubov-Valatin transformation, we obtain

$$
\begin{gathered}
\Delta_{i}=g \sum_{n}\left[u_{n i}^{\uparrow}\left(v_{n i}^{\downarrow}\right)^{*} f\left(-\varepsilon_{n}\right)+u_{n i}^{\downarrow}\left(v_{n i}^{\uparrow}\right)^{*} f\left(\varepsilon_{n}\right)\right], \\
n_{\sigma i}=\sum_{n}\left[\left|u_{n i}^{\sigma}\right|^{2} f\left(\varepsilon_{n}\right)+\left|v_{n i}^{\sigma}\right|^{2} f\left(-\varepsilon_{n}\right)\right],
\end{gathered}
$$

where $f(x)=1 /\left(e^{x / T}+1\right)$ is the Fermi-Dirac distribution function and $T$ is the temperature. Here, we set the Boltzmann constant $k_{B}$ to unity. Note that $0 \leqslant n_{\sigma i} \leqslant 1$ is the number of $\sigma$ fermions at site $i$ (number filling) such that $N_{\sigma}=\sum_{i} n_{\sigma i}$ gives the total number of $\sigma$ fermions. Equations (2)-(4) correspond to the generalization of the BdG equations [25] to the case of spin-orbit-coupled Fermi gases on optical lattices.

\section{Circulating mass currents}

Once we obtain the self-consistent solutions for the quasiparticle wave functions and the corresponding energy spectrum, it is straightforward to calculate any of the desired observables. For instance, in this paper we are interested in the quantum-mechanical probability current (mass or particle current) of $\sigma$ fermions at site $i$ defined by $\mathbf{J}_{\sigma i}=J_{\sigma i}^{x} \hat{x}+J_{\sigma i}^{y} \hat{y}$, where $J_{\sigma i}^{e}=-i t_{\sigma \sigma}^{i, i+\hat{e}}\left\langle c_{\sigma i}^{\dagger} c_{\sigma, i+\hat{e}}-\right.$ H.c. $\rangle$ is the $e$ th component. Using the Bogoliubov-Valatin transformation, we obtain

$$
\begin{aligned}
J_{\sigma i}^{e}= & -i t_{\sigma \sigma}^{i, i+\hat{e}} \sum_{n}\left[\left(u_{n i}^{\sigma}\right)^{*} u_{n, i+\hat{e}}^{\sigma} f\left(\varepsilon_{n}\right)\right. \\
& \left.+\left(v_{n i}^{\sigma}\right)^{*} v_{n, i+\hat{e}}^{\sigma} f\left(-\varepsilon_{n}\right)-\text { H.c. }\right],
\end{aligned}
$$

where we set the lattice spacing and $\hbar$ to unity. In our two-dimensional system, we expect mass currents to circulate around the central site, so that $\mathbf{J}_{\sigma i}=J_{\sigma i} \hat{\theta}$, where $\theta$ is the azimuthal angle. In addition, the local angular momentum associated with such a circulating mass current is in the $z$ direction, and its magnitude at a particular site $i$ is simply 
related to the local mass current by $L_{\sigma i}^{z}=x_{i} J_{\sigma i}^{y}-y_{i} J_{\sigma i}^{x}$, where $x_{i}$ and $y_{i}$ are the coordinates of site $i$ with respect to the center, i.e., the minimum of the trapping potential. In this paper, we are interested in the total absolute angular momentum per $\sigma$ particle, which is given by $L_{\sigma}^{z}=\left(1 / N_{\sigma}\right) \sum_{i} r_{i}\left|J_{\sigma i}\right|$.

Having established the BdG formalism, now we are ready to present our numerical solutions for the ground-state and finite-temperature phases, which are obtained by solving Eqs. (2)-(4) in a self-consistent manner.

\section{NUMERICAL RESULTS}

Our numerical calculations are performed on a twodimensional square lattice with $L=48$ lattice sites in each direction. We take $V_{0} \approx 0.014 t$ as the strength of the trapping potential, and discuss both symmetric $(\alpha=\beta)$ and asymmetric $(\alpha \neq \beta)$ SOC fields. Since we are mainly interested in lowfilling population-balanced systems, we set $N_{\uparrow}=N_{\downarrow}=125$, and study the resultant phases as a function of $g, \alpha$, and $\beta$. However, we briefly comment on the effects of high filling and population imbalance on the ground-state phases toward the end of this section.

In Fig. 1, we show the strength of the $\uparrow$ mass current $\mathbf{J}_{\uparrow i}$ at zero temperature $(T=0)$ for noninteracting $(g=0)$ systems with symmetric and asymmetric SOC, where we set $\alpha=\pi / 4$ and vary the $\beta$ parameter. We use Fig. 1(a) as our reference data in all of our discussions below. First of all, the coarse graining of the data near the trap center is a finite-size lattice effect and it does not affect our main findings. In addition, due to the timereversal symmetry, $\mathbf{J}_{\downarrow i}$ has exactly the opposite circulation for population-balanced systems and this current is not shown throughout this paper. As $\beta$ decreases from the symmetric case with $\beta=\pi / 4$ to $\beta=0$, the asymmetry between the $x$ and $y$ directions increases in such a way that the spin-flipping hopping gradually decreases to zero in the $y$ direction, and the SOC field eventually becomes purely one dimensional in the $x$ direction. We find that the mass current flows as long as the SOC field has both $x$ and $y$ components, and its strength decreases gradually as a function of increasing asymmetry of the SOC fields. Note that, since the asymmetric SOC breaks the $C_{4}$ symmetry of the square lattice, the resultant mass currents also have reduced symmetry in Figs. 1(b) and 1(c). In addition, we see that the gas expands a little with increasing asymmetry, which is due to the decrease in density of single-particle states.

In Fig. 2, we show the mass current $J_{\uparrow i}^{y}$ and filling $n_{\uparrow i}$ of $\uparrow$ fermions at $T=0$ for noninteracting systems with varying symmetric SOC strengths $\alpha=\beta$. We find that $J_{\uparrow i}$ first increases and then decreases as $\alpha=\beta$ is increased from 0 to $\pi / 2$. It is expected that the strength of the mass current rapidly increases as a function of increasing spin-flipping hopping with respect to the spin-conserving hopping, since the presence of a nonzero SOC is what allowed the mass current to form in the first place. However, as the SOC terms dominate, the chemical potential gradually drops below the band minimum, and then the system gradually crosses over to the molecular BEC side. We note that, in contrast to the no-SOC case where the formation of a two-body bound state between $\uparrow$ and $\downarrow$ fermions requires a finite interaction threshold in a two-dimensional lattice, here it can form even for arbitrarily small interactions simply by an increase in the SOC, due to
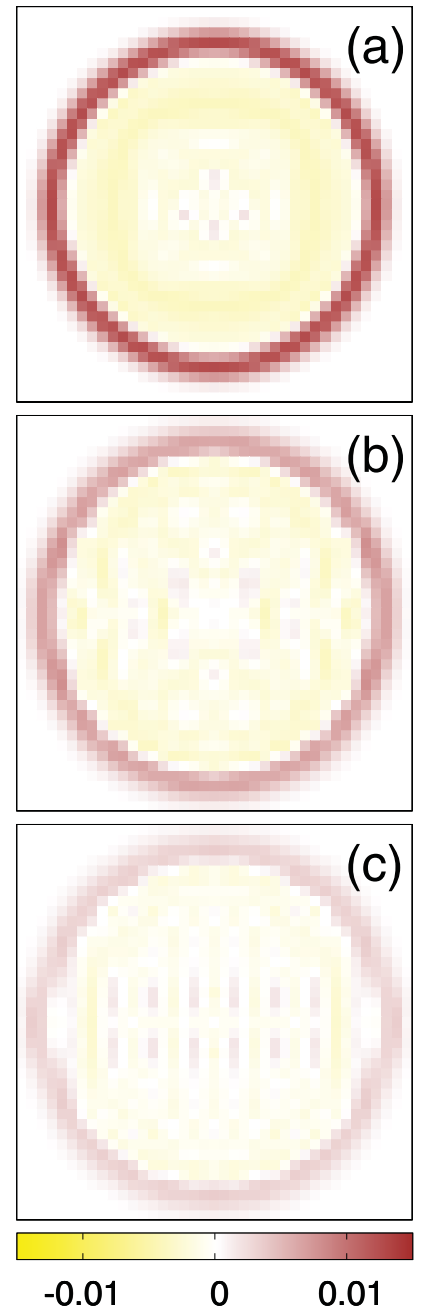

FIG. 1. (Color online) The strength of the $\uparrow$ mass current $\mathbf{J}_{\uparrow i}$ (in units of $t$ ) is shown as a function of lattice coordinates $x_{i}$ and $y_{i}$ at zero temperature $(T=0)$ for noninteracting systems $(g=0)$. Here, we consider (a) symmetric SOC where we set $\alpha=\beta=\pi / 4$, and (b),(c) asymmetric SOCs where we set $\beta$ to $\pi / 8$ in (b) and $\pi / 16$ in (c), while keeping $\alpha=\pi / 4$. The lighter, yellow (darker, red) indicates clockwise (counterclockwise) circulation. $\mathbf{J}_{\downarrow i}$ has exactly the opposite circulation and it is not shown.

the increased single-particle density of states. Therefore, it is expected that the rapid increase in the strength of the mass current is followed by a gradual decrease at larger SOC, and that the mass current eventually vanishes at sufficiently large SOC. In addition, we see that the gas shrinks with increasing SOC, which is again due to the increased density of states. Note that the ratio between the trapping potential and the effective hopping terms also increases as $\alpha=\beta$ increases from 0 to $\pi / 2$. We also calculate the absolute angular momentum per particle, $L_{\sigma}^{z}$, and find approximately $0.53,0.56$, and 0.38 (in units of $\hbar$ ), when $\alpha=\beta$ is set to $\pi / 4,5 \pi / 16$, and $7 \pi / 16$, respectively.

In Fig. 3, we show the mass current $J_{\uparrow i}^{y}$ and filling $n_{\uparrow i}$ of $\uparrow$ fermions at $T=0$ for symmetric $(\alpha=\beta=\pi / 4)$ SOC with varying interaction strength $g$. The corresponding superfluid order parameter $\left|\Delta_{i}\right|$ has an approximate peak value of 0 , $0.05 t$, and $1.4 t$ at the trap center when $g$ equals $0,1.4 t$, and 

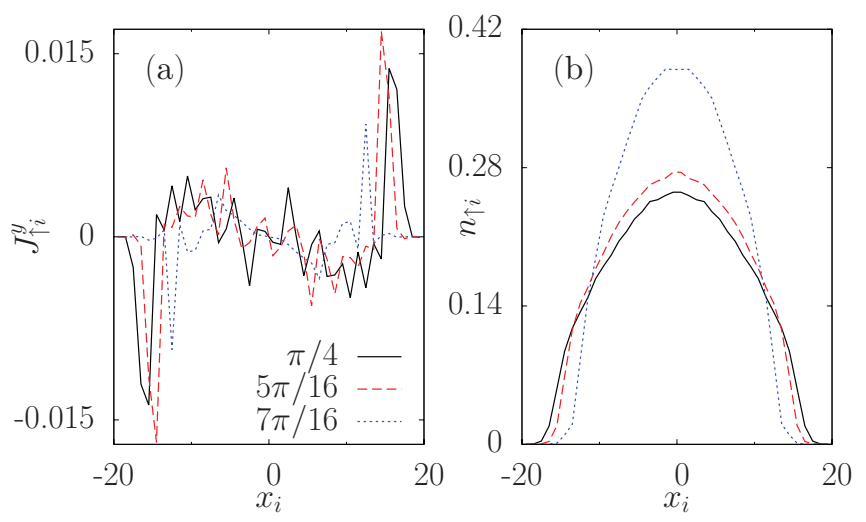

FIG. 2. (Color online) (a) The strength of the $\uparrow$ mass current $J_{\uparrow i}^{y}$ (in units of $t$ ), and (b) the filling of $\uparrow$ fermions $n_{\uparrow i}$ at $T=0$ as functions of $x_{i}$, when $y_{i}=0$. Here, the system is noninteracting, and we set $\alpha=\beta$ to $\pi / 4$ (solid black), $5 \pi / 16$ (dashed red), and $7 \pi / 16$ (dotted blue). Coarse-grained data are a finite-size lattice effect.

$4.2 t$, respectively (not shown). It is clearly seen that increasing the $g$ has qualitatively the same effect on the ground-state phases as increasing the SOC. For instance, we find that the mass current first increases and then decreases as a function of increasing $g$. In addition, the gas shrinks with increasing $g$, due to the formation of more tightly bound Cooper pairs. We again calculate the absolute angular momentum per particle, $L_{\sigma}^{z}$, and find approximately $0.53,0.49$, and 0.22 (in units of $\hbar$ ), when $g$ is set to $0,1.4 t$, and $4.2 t$, respectively. Therefore, $L_{\sigma}^{z}$ again deviates significantly from $\approx 0.5$ as a result of increased filling around the trap center.

In Fig. 4, we show the mass current $J_{\uparrow i}^{y}$ and filling $n_{\uparrow i}$ of $\uparrow$ fermions for noninteracting systems with varying temperature $T$, where we set symmetric $(\alpha=\beta=\pi / 4)$ SOC. Finite temperature excites more and more particles to higher oscillator states as a function of increasing $T$, and this naturally leads to an increase in the system size and decrease in the strength of the mass currents. This is clearly seen in the figure, where the peak value of $J_{\uparrow i}^{y}$ reduces approximately to half of its $T=0$ value when $T \simeq 0.14 t$, but with a larger width. We
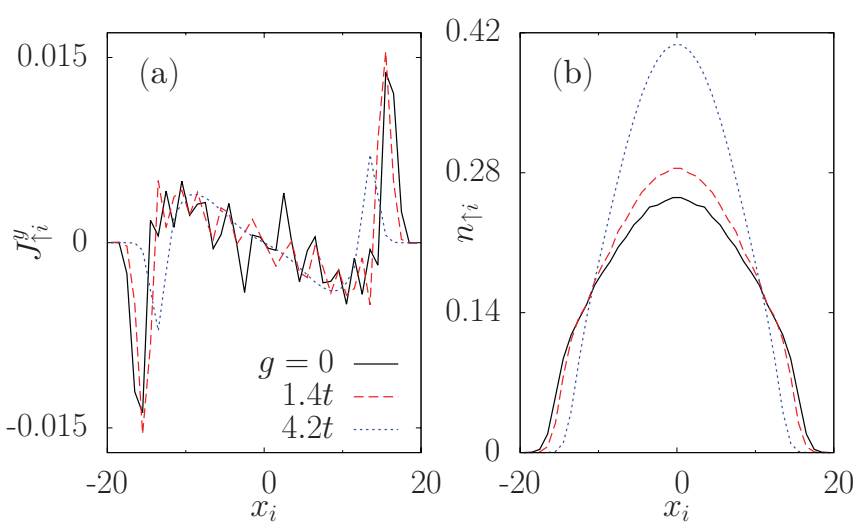

FIG. 3. (Color online) (a) The strength of the $\uparrow$ mass current $J_{\uparrow i}$ (in units of $t$ ), and (b) the filling of $\uparrow$ fermions $n_{\uparrow i}$ at $T=0$ as functions of $x_{i}$, when $y_{i}=0$. Here, the SOC is symmetric with $\alpha=\beta=\pi / 4$, and we set $g$ to 0 (solid black) $1.4 t$ (dashed red), and $4.2 t$ (dotted blue). Coarse-grained data are a finite-size lattice effect.
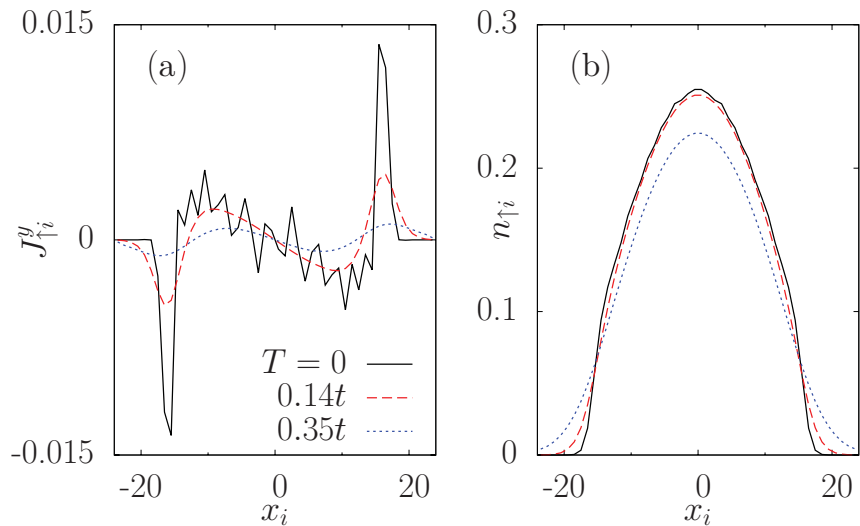

FIG. 4. (Color online) (a) The strength of the $\uparrow$ mass current $J_{\uparrow i}$ (in units of $t$ ), and (b) the filling of $\uparrow$ fermions $n_{\uparrow i}$ at finite $T$ as a function of $x_{i}$, when $y_{i}=0$. Here, the system is noninteracting and SOC is symmetric with $\alpha=\beta=\pi / 4$, and we set $T$ to 0 (solid black) $0.14 t$ (dashed red), and $0.35 t$ (dotted blue). Coarse-grained data are a finite-size lattice effect.

calculate the absolute angular momentum per particle, $L_{\sigma}^{z}$, and find approximately $0.53,0.33$, and 0.16 (in units of $\hbar$ ), when $T$ is set to $0,0.14 t$, and $0.35 t$, respectively. Therefore, $L_{\sigma}^{z}$ decreases significantly from its ground-state value $\approx 0.5$ as the temperature increases.

So far we have discussed only population-balanced systems with a low filling at the trap center, i.e., $n_{\sigma i} \lesssim 0.4$, and here we briefly comment on the effects of high filling and population imbalance on the ground-state phases [25]. First of all, due to the particle-hole symmetry of the Hamiltonian around $n_{\sigma i}=$ 0.5 , in addition to the mass-current peak circulating in the vicinity of the trap edge, an additional peak that is circulating mostly near the trap center is induced when the trap center is close to a band insulator, i.e., $n_{\sigma i} \simeq 1$. Therefore, mass currents show a double-ring structure in high-filling lattice systems. Second, the strengths of mass currents are quite robust against the effects of imbalanced populations. For instance, when the total number of $\downarrow$ fermions is reduced from $N_{\downarrow}=125$ in Fig. 1(a) to $N_{\downarrow}=20$ while keeping $N_{\uparrow}=125$ fixed, so that $n_{\uparrow} \approx 0.3$ and $n_{\downarrow} \approx 0.05$ at the trap center, the maximum currents $J_{\uparrow} \gtrsim J_{\downarrow} \approx 5 \times 10^{-3} t$ occur at about the same radial distance as the population-balanced case shown in Fig. 1(a).

\section{DISCUSSION}

Having established the qualitative behavior of spontaneously induced counterflowing mass currents in spin-orbitcoupled Fermi gases, next we argue that the origin of these currents can be understood via a direct correspondence with chiral $p$-wave superfluids and superconductors.

\section{A. Correspondence with chiral $\boldsymbol{p}$-wave systems}

The origin of spontaneously induced counterflowing mass currents near the trap edge can be understood via a direct correspondence with the $p_{x}+i p_{y}$ superfluids and superconductors, e.g., the $A$ phase of liquid ${ }^{3} \mathrm{He}$, for which it is known that a spontaneous mass current also flows near the boundary $[4,5]$. In these $p$-wave systems, the mass current 
and hence the macroscopic angular momentum are associated with the chirality of Cooper pairs. The chirality can be most easily seen by noting that the chiral $p$-wave order parameter $\Delta_{\mathbf{k}} \propto(\hat{x} \pm i \hat{y}) \cdot \mathbf{k}$, where $\mathbf{k}$ is the relative momentum of a Cooper pair, is an eigenfunction of the orbital angular momentum with eigenvalue $\pm \hbar$. This mechanism explains our findings since it can be shown that the order parameter of spin-orbit-coupled Fermi gases with Rashba-type SOC and $s$-wave contact interactions has chiral $p$-wave symmetry. To see this one needs to transform the Hamiltonian to the helicity basis, i.e., the pseudospin is no longer a good quantum number in the presence of SOC, and the spin direction is either parallel or antiparallel to the direction of the in-plane momentum in the \pm helicity basis. In the helicity-basis representation, it becomes clear that only intraband Cooper pairing occurs between fermions with the same helicity and that the order parameter for \pm helicity pairing has $p_{x} \mp i p_{y}$ symmetry [12].

Although the basic mechanisms in chiral $p$-wave systems and spin-orbit-coupled Fermi gases share some similarities with respect to spontaneously induced edge currents, they also differ in fundamental ways. For instance, in contrast to the chiral $p$-wave systems where the formation of Cooper pairs and hence the mass current requires an interacting system, in spin-orbit-coupled Fermi gases currents flow even in the absence of interactions. In addition, the chiral $p$-wave systems belong to the topological class of integer quantum Hall systems, and since these systems both break timereversal symmetry, they exhibit spontaneous edge currents circulating along a particular direction. However, spin-orbitcoupled Fermi gases preserve time-reversal symmetry just like quantum spin Hall systems, and therefore they both exhibit spontaneously induced counterflowing $\uparrow$ and $\downarrow$ edge currents. Next, we comment on the experimental realization of spontaneously induced edge currents in atomic systems.

\section{B. Experimental realization}

In comparison to bulk (center) effects, there is no doubt that the edge effects are more difficult to observe in trapped atomic systems. This is because as the local density decreases from the central region to the edges, it gets harder to probe local properties. In addition, the local critical superfluid temperature eventually drops below the temperature of the system near the edges, and the superfluidity is also lost starting from the edges inward. One way to partially overcome such problems could be to load the optical lattice potential with higher particle fillings such that the trap center is close to a band insulator, i.e., unit filling. In this case, an additional mass-current peak circulates mostly near the trap center (see above), and such a local current could be easier to probe with current experimental capabilities.

Measurement of the angular momentum of rotating atomic systems has so far been achieved only indirectly, by observing the shift of the radial quadrupole modes. While this technique was initially used for rotating atomic BECs [26,27], it has recently been applied to rotating fermionic superfluids in the BCS-BEC crossover [28]. We believe a similar technique could be used for measuring the intrinsic angular momentum of spin-orbit-coupled Fermi gases, which may provide indirect evidence for counterflowing mass currents. In addition, we note that a realistic scheme has recently been proposed to detect topological edge states in an optical lattice under a synthetic magnetic field [29]. This proposal is based on a generalization of Bragg spectroscopy that is sensitive to angular momentum.

\section{CONCLUSIONS}

To conclude, we showed that the presence of Rashba-type SOC spontaneously induces counterflowing $\uparrow$ and $\downarrow$ mass currents. While these currents have a single peak that is circulating mostly near the trap edge in low-filling lattice systems, an additional peak that is circulating mostly near the trap center is also induced in high-filling lattice systems, which exhibit a double-ring structure. We note that our results for the low-filling lattice systems are directly applicable to dilute systems (without the optical lattice potential), for which we expect qualitatively similar behavior. These currents flow even in noninteracting systems, and they are quite robust against the effects of imbalanced populations and/or asymmetric SOC in the $x$ and $y$ directions. However, their strength decreases toward the molecular BEC limit, which can be achieved by increasing either the SOC or the interaction strength. We argued that the origin of spontaneously induced counterflowing mass currents in spin-orbit-coupled Fermi gases can be understood via a direct correspondence with chiral $p$-wave superfluids and superconductors, but with some fundamental differences.

\section{ACKNOWLEDGMENTS}

This work is supported by the Marie Curie IRG Grant No. FP7-PEOPLE-IRG-2010-268239, the TÜBITAK Career Grant No. 3501-110T839, and TÜBA-GEBIP. E.D. is also supported by a TÜBITAK-2215 Ph.D. Fellowship.
[1] For a review, see A. J. Leggett, Quantum Liquids: Bose Condensation and Cooper Pairing in Condensed-Matter Systems (Oxford University Press, Oxford, 2006), Appendix 6A.

[2] For a review, see A. P. Mackenzie and Y. Maeno, Rev. Mod. Phys. 75, 657 (2003).

[3] J. P. Gaebler, J. T. Stewart, J. L. Bohn, and D. S. Jin, Phys. Rev. Lett. 98, 200403 (2007).

[4] M. Stone and I. Anduaga, Ann. Phys. (NY) 323, 2 (2008).
[5] T. Mizushima, M. Ichioka, and K. Machida, Phys. Rev. Lett. 101, 150409 (2008).

[6] M. Inguscio, W. Ketterle, and C. Salomon, Ultra-Cold Fermi Gases, Proceedings of the International School of Physics "Enrico Fermi," Course CLXIV, Varenna (IOS, Amsterdam, 2008).

[7] Y. J. Lin, R. L. Compton, A. R. Perry, W. D. Phillips, J. V. Porto, and I. B. Spielman, Phys. Rev. Lett. 102, 130401 (2009). 
[8] Y.-J. Lin et al., Nature (London) 471, 83 (2011).

[9] Pengjun Wang et al., e-print arXiv:1204.1887.

[10] M. Gong, S. Tewari, and C. Zhang, Phys. Rev. Lett. 107, 195303 (2011); G. Chen, M. Gong, and C. Zhang, Phys. Rev. A 85, 013601 (2012).

[11] J. P. Vyasanakere, S. Zhang, and V. B. Shenoy, Phys. Rev. B 84, 014512 (2011).

[12] Z. Q. Yu and H. Zhai, Phys. Rev. Lett. 107, 195305 (2011); H. Zhai, Int. J. Mod. Phys. B 26, 1230001 (2012).

[13] Hui Hu, L. Jiang, X.-J. Liu, and Han Pu, Phys. Rev. Lett. 107, 195304 (2011); L. Jiang, X.-J. Liu, H. Hu, and H. Pu, Phys. Rev. A 84, 063618 (2011).

[14] M. Iskin and A. L. Subaşı, Phys. Rev. Lett. 107, 050402 (2011); Phys. Rev. A 84, 043621 (2011).

[15] W. Yi and G.-C. Guo, Phys. Rev. A 84, 031608(R) (2011).

[16] Li Han and C. A. R. Sá de Melo, Phys. Rev. A 85, 011606(R) (2012).

[17] L. He and X. G. Huang, Phys. Rev. Lett. 108, 145302 (2012).
[18] S. K. Ghosh, J. P. Vyasanakere, and V. B. Shenoy, Phys. Rev. A 84, 053629 (2011).

[19] B. Huang and S. Wan, e-print arXiv:1109.3970; X. Yang and S. Wan, Phys. Rev. A 85, 023633 (2012)

[20] J. Zhou, W. Zhang, and W. Yi, Phys. Rev. A 84, 063603 (2011).

[21] J. N. Zhang, Y. H. Chan, and L. M. Duan, e-print arXiv:1110.2241.

[22] K. Zhou and Z. Zhang, Phys. Rev. Lett. 108, 025301 (2012).

[23] R. Liao, Y. Yi-Xiang, and W.-M. Liu, Phys. Rev. Lett. 108, 080406 (2012).

[24] M. Iskin, Phys. Rev. A 85, 013622 (2012).

[25] M. Iskin and C. J. Williams, Phys. Rev. A 78, 011603(R) (2008).

[26] F. Chevy, K. W. Madison, and J. Dalibard, Phys. Rev. Lett. 85, 2223 (2000)

[27] P. C. Haljan, B. P. Anderson, I. Coddington, and E. A. Cornell, Phys. Rev. Lett. 86, 2922 (2001).

[28] S. Riedl, E. R. Sanchez Guajardo, C. Kohstall, J. H. Denschlag, and R. Grimm, Phys. Rev. A 79, 053628 (2009).

[29] N. Goldman et al., e-print arXiv:1203.1246. 